\title{
Final Technical Report for Award DE-FG02-07ER64429
}

Title: Collaborative Research: Regional climate-change projections through next-generation empirical and dynamical models

Principal Investigator:

Padhraic J. Smyth (University of California, Irvine)

Award Number: DE-FG02-07ER64429

Award period: 07/15/07-07/14/10

This project was a continuation of previous work under DOE CCPP funding in which we developed a twin approach of non-homogeneous hidden Markov models (NHMMs) and coupled ocean-atmosphere (O-A) intermediate-complexity models (ICMs) to identify the potentially predictable modes of climate variability, and to investigate their impacts on the regional-scale. We have developed a family of latentvariable NHMMs to simulate historical records of daily rainfall, and used them to downscale seasonal predictions. We have also developed empirical mode reduction (EMR) models for gaining insight into the underlying dynamics in observational data and general circulation model (GCM) simulations. Using coupled O-A ICMs, we have identified a new mechanism of interdecadal climate variability, involving the midlatitude oceans' mesoscale eddy field and nonlinear, persistent atmospheric response to the oceanic anomalies. A related decadal mode is also identified, associated with the oceans' thermohaline circulation.

The goal of the continuation was to build on these ICM results and NHMM/EMR model developments and software to strengthen two key pillars of support for the development and application of climate models for climate change projections on time scales of decades to centuries, namely: (a) dynamical and theoretical understanding of decadal-to-interdecadal oscillations and their predictability; and (b) an interface from climate models to applications, in order to inform societal adaptation strategies to climate change at the regional scale, including model calibration, correction, downscaling and, most importantly, assessment and interpretation of spread and uncertainties in multi-model ensembles.

Our main results from the grant consist of extensive further development of the hidden Markov models for rainfall simulation and downscaling specifically within the non-stationary climate change context together with the development of parallelized software; application of NHMMs to downscaling of rainfall projections over India; identification and analysis of decadal climate signals in data and models; and, studies of climate variability in terms of the dynamics of atmospheric flow regimes. Each of these project components is elaborated on below, followed by a list of publications resulting from the grant.

\section{Hidden Markov models}

\section{a) Methodological development}

We have developed hidden Markov models (HMMs) to model mutivariate fields of daily rainfall via a hidden state variable with Markov dynamics. The model parameters include the Markov transition matrix 
on states, and state-dependent mixture distributions for the rainfall observations. An extension allows the Markov state dynamics to be driven by a set of inputs from an exogenous input variable. We have found that these types of hidden Markov models can provide parsimonious and interpretable characterizations of the spatio-temporal dynamics of regional precipitation processes, where each of the estimated states corresponds to a persistent and recurrent "weather state" with a distinct spatial precipitation pattern.

An important question concerns nonstationarity, and have used bootstrap techniques to assess this in precipitation patterns of the NCAR CCSM3 and ECHAM5 GCM climate change projections (A2 scenario) over India. We fit two HMMs to precipitation data from two different 20-year time-periods: 1981-2000 and 2081-2100. In both the CCSM3 and ECHAM5 GCM projections, the results clearly indicate that there is substantial non-stationarity present between the two time-periods, i.e., that for a large fraction of the HMM parameters there are changes that can only be accounted for by non-stationarity in the underlying precipitation process. The main characteristic of the changes is that the wettest state (of the $\mathrm{K}=3$ states) occurs less frequently in 2081-2100 compared to 1981-2000, but when it does occur it produces higher precipitation amounts on average. Seasonal-average increases in rainfall in both model over northern India, are thus factorized by the HMM into more heavy rainfall events, but on fewer rainy days.

The estimation of $N=500$ HMMs on $N$ simulated data sets is very computationally intensive, particularly since to fit an HMM model to each data set the iterative expectation-maximization (EM) algorithm must be run multiple times from different initial conditions so as to avoid local maxima in the likelihood surface. To speed up this process we have implemented a version of our EM code that can parallelize the different restarts across different processes - we have implemented this on a multi-core Linux machine with 8 processors and obtained a factor of 7 speed-up in run-time for $K=3$ states and 15 restarts (yielding a parallelization efficiency of approximately 85\%), reducing the run-time from about 18 hours to about 2.5 hours for an $N=500$ bootstrap simulation run.

We have developed methods that allow rainfall amounts to vary as a function of thermodynamic factors such as humidity, while independently allowing the NHMM states to vary in response to atmospheric dynamics. This is required for climate-change scenarios, since it allows a model (a) to decrease the probability of precipitation occurrence in a given region (by decreasing the probability of visiting high occurrence states in the NHMM), and (b) to simultaneously increase the intensity of simulated rainfall from the model, resulting in overall increases in mean precipitation amounts and decreases in occurrence. We have developed a statistical framework based on generalized linear models (GLMs) that extend our earlier NHMM modeling framework to allow mean precipitation intensity (at a particular station, in a given state) to vary in response to a daily input variable such as humidity. Algorithms based on the expectation-maximization (EM) framework have been developed and tested for parameter estimation of such joint NHMM-GLM models, using both observed 20th century and GCM data. Models fit to 20th century data can then be run with 21st century GCM inputs to produce downscaled rainfall simulations at daily temporal scales and local regional scales.

\section{b) Application of NHMMs to downscaling of rainfall projections}


Nonhomogeneous hidden Markov models (NHMMs) have potential utility not only as diagnostic tools, but, perhaps more critically, in the downscaling of regional climate change projections. To this end we have developed and refined an NHMM modeling framework wherein large-scale climate change signals from an ensemble of general circulation models (GCMs) are optimally combined with data from a dense observational network to produce projections for the Indian summer monsoon that are both constrained by the observed spatiotemporal variability and conditioned on GCM-based climate change information. In this framework the state space of observed rainfall is preserved, but population statistics for its various basins of attraction are conditioned by climatic variations from the GCM ensemble. Ensemble mean fields are utilized for the projections, while intermodel dispersion serves to characterize uncertainty owing to structural model variations. The method bypasses both daily rainfall distributional biases due to uncertain GCM parameterization schemes and GCM biases on fine spatial scales arising from the finite resolution of these models. The observational network is now defined on a uniform grid covering the entire monsoon zone, permitting improved characterization of the large-scale weather states that condition NHMM parameters.

The work originates with the diagnostic study presented in Greene et al. (2008), and continues with Greene et al. (2011).

\section{Decadal and multi-decadal natural climate variability in observations, idealized climate models, and General Circulation Model (GCM) simulations}

Our work proceeded in two inter-related directions: (1) Identifying and analyzing decadal climate signals in data and models; and (2) Studying the dynamics of atmospheric regimes -- anomalously persistent patterns that appear to preferentially respond to ocean-induced decadal forcing and potentially result in the predictable coupled modes. Our major statistical analysis tools included the Empirical Model Reduction (EMR) method (Kravtsov et al. 2005; Kondrashov et al. 2005) for fitting a nonlinear stochastic model to various climatic data sets, as well as the multivariate signal-detection techniques such as Empirical Orthogonal Function (EOF) analysis (Preisendorfer 1988) and Multi-channel Singular Spectrum Analysis (M-SSA: Ghil et al. 2002). We also ran and analyzed simulations of prototype dynamical models, including high horizontal resolution ocean/atmosphere quasi-geostrophic models in idealized geometries or stripped-down versions of GCM models to achieve better understanding of processes underlying decadal climate variability and its effect on atmospheric regimes. Our analyses covered the issues related to both global climate variability, as well as to its regional manifestations; the various regions of focus emphasized in the particular studies summarized below included the North Atlantic and North Pacific oceans, Tropical Pacific Ocean, Southern Ocean, and the continental US region:

(1) Empirical Model Reduction (EMR). (a) EMR review book chapter: Kravtsov et al. (2009a); (b) EMR as a major tool for identification and analysis of nonlinear regimes of atmospheric circulation: Kravtsov et al. (2009b) -- application to an atmospheric GCM; Kondrashov et al. (2010), Schwartz and Kravtsov (2010) -- application to an idealized atmospheric model (QG3); (c) EMR as an efficient stochastic parameterization scheme: Strounine et al. (2010), Culina et al. (2010); (d) EMR-related ENSO modeling: Chekroun et al. (2010); Kravtsov (2010). 
(2) Statistical analysis of climatic data sets. (a) Observed and GCM simulated global climates --Kravtsov and Spannagle (2008), Kravtsov et al. (2008b), Jamison and Kravtsov (2010), Wyatt et al. (2011); (b) Regional climate in relation to larger-scale climate and weather variations: Hanrahan et al. $(2010,2011)$-climate-related variability of Lake Michigan and Huron levels, Dharshana et al. (2011) -- climate and particulate-matter air pollution over the US.

(3) Idealized dynamical modeling to address effects of oceanic mesoscale turbulence on large-scale decadal climate variability: Kravtsov et al. (2008a,b) -- Northern Hemisphere's middle latitudes, Hogg et al. (2009), Kravtsov et al. (2011) -- Southern Ocean.

The complete citations to the above papers are listed in section 3, and their technical summaries follow immediately below:

Kravtsov et al. (2009a): We present here a methodology for constructing intermediate climate models based almost entirely on the observed evolution of selected climate fields, without reference to dynamical equations that may govern this evolution; these models parameterize unresolved processes as multivariate stochastic forcing.

Kravtsov et al. (2009b): Simulations using an idealized, atmospheric general circulation model (GCM) subjected to various thermal forcings are analyzed via a combination of probability density function (PDF) estimation and spectral analysis techniques. Statistically significant deviations from the linear stochastic null hypothesis arise in the form of a few anomalously persistent, or statistically nonlinear, flow patterns, which occupy particular regions of the model's phase space.

Kondrashov et al. (2011): Phase-space tendencies of an atmospheric model are attributed to the nonlinear interactions between "resolved" modes in the empirical mode reduction (EMR) approach, rather than to the effect of the "noise" associated with unresolved modes.

Peters et al. (2011): Numerous previous studies addressed mid-latitude atmospheric flow patterns, or regimes, that persist for periods of time exceeding typical lifetimes of weather systems (that is, a few days). In this study, output of a realistic atmospheric model is analyzed to examine relationships between regionally intensified and predominantly zonally symmetric regimes.

Strounine et al. (2010): Low-frequency variability (LFV) of the atmosphere refers to its behavior on time scales of 10-100 days, longer than the life cycle of a mid-latitude cyclone but shorter than a season. This behavior is still poorly understood and hard to predict. The present study compares various model reduction strategies that help in deriving simplified models of LFV.

Culina et al. (2010): In this study, a parameterisation method for use in complex climate models was developed from limiting theorems that predict the effective slow dynamics in systems with coupled slow and fast variables using an example of quasi-geostrophic model of midlatitude low-frequency variability.

Chekroun et al. (2010): A new method of climate forecasting that uses general empirical stochastic models (EMRs) is developed. This method is divided into selecting noise samples (snippets) from the past 
noise and use of these snippets to drive the system from the current observations into the future. For an empirical ENSO model, the method produces significantly better skill compared to that of the base EMR model.

Kravtsov (2011): This paper assesses potential predictability of decadal variations in the El Nino/Southern Oscillation (ENSO) characteristics by constructing and performing simulations using an empirical nonlinear model of the Nino-3 index. The model predicts a net reduction in ENSO activity by the year 2020. Retroactive ENSO-frequency forecasts possess a useful skill at decadal lead times.

Kravtsov and Spannagle (2008): This study identifies interdecadal natural climate variability in global surface temperatures by subtracting, from the observed temperature evolution, multi-model ensemble mean based on the CMIP3 dataset. The resulting signal resembles the so-called Atlantic Multidecadal Oscillation (AMO).

Kravtsov et al. (2008b): We show that the observed zonally averaged jet in the Northern Hemisphere atmosphere exhibits two spatial patterns with broadband variability in the decadal and inter-decadal range; these patterns are consistent with an important role of local, mid-latitude ocean-atmosphere coupling. A key aspect of this behaviour is the fundamentally nonlinear bi-stability of the atmospheric jet's latitudinal position, which enables relatively small sea-surface temperature anomalies associated with ocean processes to affect the large-scale atmospheric winds.

Jamison and Kravtsov (2010): This study evaluates the ability of the CMIP3 climate models to simulate intrinsic decadal variations detected in the observed North Atlantic sea-surface temperature (SST) record via multi-channel Singular Spectrum Analysis (M-SSA). Many of the CMIP3 20th-century simulations are characterized by quasi-oscillatory behavior within one or more of the three observationally motivated frequency bands; however, only a fraction of these models also capture the spatial patterns of the observed signals.

Wyatt et al. (2011): Multidecadal signal with a periodicity of 50-80 yr has been previously identified in a variety of proxy and instrumental records of the Northern Hemisphere's and global climate; it bears the name of the Atlantic Multidecadal Oscillation (AMO). By analyzing lagged covariance structure of a network of climate indices, this study details the ways the AMO signal propagates throughout the Northern Hemisphere via a sequence of atmospheric and lagged oceanic teleconnections.

Hanrahan et al. (2009): This study identifies decadal cycles of Great Lakes' water levels in an instrumental record. It revealed 8 and 12-yr period oscillations, whose time scales match those of largescale climatic signals previously found in the North Atlantic. Furthermore, water budget analysis argues that the North Atlantic decadal climate modes translate to the lake levels primarily through precipitation and its associated runoff.

Hanrahan et al. (2010): Lake Michigan levels are analyzed after outflow-related damping effects were removed, increasing the transparency of the lake level fluctuations and potential climate connections. This filtering exposes a large oscillation, which is connected to the Atlantic Multidecadal Oscillation (AMO), 
as well as previously identified decadal signals. Summertime evaporation rates have more than doubled since 1980 and drive some of the recent lake-level changes.

Dharshana et al. (2011): This study establishes connections between day-to-day variability of meteorological parameters and that of the particulate-matter (PM) air pollution. The main effect of synoptic weather systems on day-to-day variability of PM air pollution consists of formation of a highpollution area behind propagating anticyclones and a low-pollution region in the wake of cyclones. This $\mathrm{PM}$ variability accounts for as much as $50 \%$ of the total $\mathrm{PM}$ variance in the midwest region throughout the year.

Kravtsov et al. (2008a): Previous studies have treated atmospheric intrinsic variability as a linear stochastic process modified by a deterministic coupling to the ocean. We here represent this variability in terms of irregular transitions between two anomalously persistent, high-latitude and low-latitude jetstream states. A nearly analytical approximation of the corresponding conceptual coupled model is used to study the sensitivity of this behavior to key model parameters.

Hogg et al. (2009): Small-scale variation in wind stress due to ocean-atmosphere interaction within the atmospheric boundary layer alters the Ekman pumping driving the double-gyre circulation of the ocean. A high-resolution ocean model coupled to a dynamic atmospheric mixed layer demonstrates that this phenomenon significantly modifies the large-scale ocean circulation.

Kravtsov et al. (2011): The CMIP3 model ensembles were found to overestimate long-term changes in the surface-temperature gradient in the Southern Ocean. This is due to the lack of mesoscale turbulence in relatively coarse-resolution CMIP3 models, combined with underestimated increase of the zonal winds in these models.

\section{List of publications acknowledging this grant}

Camargo, S. J., A. W. Robertson, A. G. Barnston, and M. Ghil, 2008: Clustering of eastern North Pacific tropical cyclone tracks: ENSO and MJO effects. G-Cubed 9, Q06V05, doi:10.1029/2007GC001861.

Chekroun, M., D. Kondrashov, and M. Ghil, 2010: Using past noise in forecasting: Pathwise linear response and ENSO. To be submitted to PNAS.

Culina, J., S. Kravtsov, and A. Monahan, 2010: Stochastic parameterisation schemes for use in realistic climate models. J. Atmos. Sci., 68, 284-299.

Dharshana, K. G. T., S. Kravtsov, and J. D. W. Kahl, 2011: The relationship between daily weather conditions and particulate-matter air pollution over the US. J. Geophys. Res.-Atmos., J. Geophys. Res., 115, D24219, doi:10.1029/2010JD014852.

Feliks, Y., M. Ghil, and A. W. Robertson, 2010: Oscillatory Climate Modes in the Eastern Mediterranean and Their Synchronization with the North Atlantic Oscillation. J. Climate, 23, 4060-4079. 
Feliks, Y., M. Ghil, and A. W. Robertson, 2011: The atmospheric circulation over the North Atlantic as induced by the SST field. J. Climate, in press.

Greene, A.M., A. W. Robertson and S. Kirshner, 2008: Analysis of Indian monsoon daily rainfall on subseasonal to multidecadal time scales using a hidden Markov model, Q. J. Royal Met. Soc., (134) 633, 875-887.

Greene, A.M., A. W. Robertson, P. Smyth, and S. Triglia, 2011: Downscaling projections of Indian monsoon rainfall using a nonhomogeneous hidden Markov model, Q. J. Royal Met. Soc., 137, 347-359.

Hanrahan, J. L., S. Kravtsov, and P. J. Roebber, 2009: Quasi-periodic decadal cycles in levels of lakes Michigan and Huron. Great Lakes Res.,35, 30-35.

Hanrahan, J. L., S. Kravtsov, M. Ghil, and P. Roebber, 2010: Connecting past and present climate variability to the water levels of Lakes Michigan and Huron. Geophys. Res. Lett., 37, L01701, doi:10.1029/2009GL041707.

Hanrahan, J. L., P. Roebber, and S. Kravtsov, 2011: Attribution of decadal-scale water-level trends in Michigan-Huron system. J. Geophys. Res. Atmos., submitted.

Hogg, A., W. K. Dewar, P. Berloff, S. Kravtsov, and D. K. Hutchinson, 2009: The effects of mesoscale ocean-atmosphere coupling on the large-scale ocean circulation. J. Climate, 22, 4066-4082.

Jamison, N., and S. Kravtsov, 2010: Decadal variations of North Atlantic sea-surface temperature in observations and CMIP3 simulations. J. Climate, 23, 4619-4636.

Kondrashov, D., S. Kravtsov, and M. Ghil, 2011: Signatures of nonlinear dynamics in an idealized atmospheric model. J. Atmos. Sci., 68, 3-12.

Kravtsov, S., 2011: An empirical model of decadal ENSO variability. J. Climate, sub judice.

Kravtsov, S., M. Ghil, and D. Kondrashov, 2009a: Empirical Model Reduction and the Modeling Hierarchy in Climate Dynamics and the Geosciences. Stochastic Physics and Climate Modeling, T. Palmer and P. Williams, Eds., Cambridge University Press, pp. 35-72.

Kravtsov, S., Hoeve, J. E. T., S. B. Feldstein, S. Lee, and S.-W. Sun, 2009b: The relationship between statistically linear and nonlinear feedbacks and zonal-mean flow variability in an idealized climate model. J. Atmos. Sci., 66, 353-372.

Kravtsov, S., and C. Spannagle, 2008: Multi-decadal climate variability in observed and simulated surface temperatures. J. Climate, 21, 1104-1121.

Kravtsov, S., W. K. Dewar, M. Ghil, J. C. McWilliams, and P. Berloff, 2008a: A mechanistic model of mid-latitude decadal climate variability. Physica D, 237, 584-599, doi:10.1016/j.physd.2007.09.025. 
Kravtsov, S., W. K. Dewar, P. Berloff, J. C. McWilliams, and M. Ghil, 2008b: North Atlantic climate variability in coupled models and data. Nonlin. Proc. Geophys., 15, 13-24.

Kravtsov, S., I. Kamenkovich, A. Hogg, and J. M. Peters, 2011: On the mechanisms of late 20th century sea-surface temperature trends over Antarctic Circumpolar Current. J. Geophys. Res. Oceans, submitted.

Moron, V., A. W. Robertson and R. Boer, 2009: Spatial coherence and seasonal predictability of monsoon onset over Indonesia. J. Climate 22, 840-850.

Moron, V., A. Lucero, F. Hilario, B. Lyon, A. W. Robertson and D. DeWitt, 2010: Spatio-temporal variability and predictability of summer monsoon onset over the Philippines. Climate Dynamics, 33, 1159-1177

Moron, V., A. W. Robertson and J.-H. Qian, 2010: Local versus large-scale characteristics of monsoon onset and post-onset rainfall over Indonesia. Climate Dynamics, 34, 281-299.

Moron, V., A. W. Robertson, and M. Ghil, 2011: Impact of the modulated annual cycle and intraseasonal oscillation on daily-to-interannual rainfall variability across monsoonal India. Climate Dynamics, submitted.

Peters, J., S. Kravtsov, and N. Schwartz, N., 2011: Zonally symmetric and regionally intensified regimes of atmospheric circulation: Persistence, transitions and predictability. J. Atmos. Sci., sub judice.

Robertson, A. W., V. Moron, and Y. Swarinoto, 2009: Seasonal predictability of daily rainfall statistics over Indramayu district, Indonesia. Int. J. Climatology, 29, 1449-1462.

Strounine, K., S. Kravtsov, D. Kondrashov, and M. Ghil, 2010: Reduced models of atmospheric lowfrequency variability: Parameter estimation and comparative performance. Physica D, 239, 145-166, doi:10.1016/j.physd.2009.10.013.

Wyatt, M., S. Kravtsov, and A. A. Tsonis, 2011: Atlantic Multidecadal Oscillation and Northern Hemisphere's climate variability. Climate Dyn., accepted.

Verbist, K., A. W. Robertson, W. M. Cornelis, D. Gabriels, 2010: Seasonal predictability of daily rainfall characteristics in central-northern Chile for dry-land management. J. Appl. Meteor. Climatol., 49, 19381955.

Yoo, J. H., A. W. Robertson, and I.-S. Kang, 2010: Analysis of Intraseasonal and Interannual variability of the Asian summer monsoon using a Hidden Markov Model. J. Climate, 23, 5498-5515. 